\title{
Adaptive E-Learning GRID Platform
}

\author{
N.Bogonikolos, K. Giotopoulos ${ }^{(\text {and } 3)}$ and K.Votis ${ }^{(\text {and } 3)}$ \\ ZEUS Consulting S.A., \\ 93 Riga Feraiou str., 26221 Patras, Greece ${ }^{1}$. \\ M. Chrysostalis \\ Western Greece Development Center \\ 138 Agiou Andreou Str., 26222 Patras, Greece ${ }^{2}$ \\ S. Likothanassis \\ Department of Computer Engineering and Informatics \\ 26110 Patras, Greece ${ }^{3}$ and Computer Technology Insitute \\ 3 Kolokotroni Str, 26221 Patras, Greece
}

\begin{abstract}
Many analysts believe that more and more organizations are looking to introduce e-learning as a way to solve critical business problems and as a vital component of an integrated approach to their training solutions. So we are introducing our next generation, e-learning grid platform to meet the evolving needs of the market, which include technology, content and services. This platform will define a new mode of service interaction and application creation. It proposes a user-friendly method for deploying interactive (mobile) devices and for accessing new and innovative added value services. The specific infrastructure will provide the technology necessary to access, arrange, manage and make available e-learning services and applications, for both individual end users and business.
\end{abstract}

Keywords: e-learning, grid technologies, platforms, e-learning services, grid architecture, mobile, Intelligent agents

\section{INTRODUCTION}

Fifty years ago, technical know-how was a relatively rare commodity with as few as 20 percent of all jobs in the United States requiring skilled workers. According to a study by PricewaterhouseCooper's National High Tech Group, that number has risen sharply and will reach 85 percent by 2005 .

Everywhere you look you see basic jobs that demand technological sophistication. Shipping clerks and drivers input data into hand-held devices. Assembly line workers interface with complex supply chain and factory floor systems. Traditional work processes have quickly become the province of e-business applications. At the very least, the average employee has to be able to log on to a computer, access the Internet, and navigate around basic applications to perform day-to-day functions.

With the learning demands placed on the modern workforce, it's no surprise that 70 percent of CEOs consider finding and retaining skilled workers a serious concern. And its not just training employ-trained, personnel have to be regularly retrained or their knowledge becomes obsolete. Most analysts agree that as much as 50 percent of a person's skills and know-how becomes outdated within three to five years. And in sectors such as IT, the half-life is even shorter. That's why so many organizations are turning away from traditional education models and embracing e-learning. E-learning offers a solution to this dilemma by making courses and data available when and where the employee needs it.

So almost all of the organizations trying to find solutions in order to implement e-learning services by using e-learning portals, virtual classrooms, web applications and many others technologies. [12] 
The proposed platform will embark on the future of services. Our main objectives are to design and implement a flexible communication and information platform, which enables users, particularly computer illiterate ones, to take full advantage of the benefits provided by modern information technology through a new system of service provisioning.

Europe is well on its way into the information society, and facing the challenges and opportunities of the global market place. Yet, recent research indicates that only around 30 per cent of the European population are making use of the internet. It should be clear that Europe cannot afford to exclude such a large proportion of its population from the future.

Against this background we propose a platform that will provide the basis for user-friendly access to e-learning information and communication services. Easily adaptable and composable, will enable both business and private users, whether they are mobile or stationery, to benefit from services and applications provided through the internet.

The current market for the distribution of services and information is limited to web based services or complicated programs that manage information. The specific platform is not limited as a web service, but encompasses both the on and the offline management of information, interconnected via the Internet, hence piecing together existing services and offering more. It will address the complex nature of information management and service provisioning by introducing agent-based activities which will allow the user a specify approach. This approach will allow a user to introduce a request without the laborious task of controlling its processes. The user needs only to wait for a response to the request, he can move on to other tasks and return later to retrieve the response.

Firstly we are going to describe the current situation about some innovative services and techniques that have to be used in order to develop an innovative and effective e-learning platform. Also we are going to make an introduction to Grid Technologies in order to take the consideration that Grids is the new future technology for distributed applications. Secondly we will present the architecture and the functionalities of this e-learning platform.

\section{CURRENT TECHNICAL SITUATION AND INNOVATION}

\subsection{Composable Services}

Over the last couple of years, composable services have become a hot topic in a variety of domains. The most popular application categories include 'active networks', 'information collection', and 'e-business'; the latter, in particular, is steering several research efforts towards frameworks for the description, discovery, organization and execution of services in a distributed and adaptive fashion.

Indeed, this research area is lively and several specifications and proposal standards are competing in the arena. XML is the leading technology for services description (WSDL) and data exchange. The SOAP protocol is gaining consensus as the ubiquitous transport protocol for unidirectional message exchange, but there are still several open issues (e.g. security). In the service discovery area there are some specifications based on registries that organize advertised services into categories (e.g. UDDI), but more evolved solutions are desired. [9]

Finally, composition and evolution activities are still not mature, especially among heterogeneous providers and about transactional properties. This big picture shows how, in the near future, frameworks for the dynamic and adaptive services discovery, composition and execution will be available. In this context e-learning grid platform can provide an important contribution in several directions. First, bringing simplicity to users (usability area) as a key building block for a wide adoption of these technologies (currently, mainly large enterprises are pushing this research, especially in the area of $b 2 b$ ). Then, growing up a model for quality of service specification as an essential part of user-device and device-device interactions. Finally, researching for a new framework targeted for the dynamic discovery, composition and evolution of heterogeneous services freeing the user from all issues related to "how-to-do". 


\subsection{GRID Architecture}

A Grid is a collection of distributed computing resources available over a local or wide area network that appear to an end user or application as one large virtual computing system. The vision is to create virtual dynamic organizations through secure, coordinated resource-sharing among individuals, institutions and resources. Grid computing is an approach to distributed computing that spans not only locations but also organizations, machine architectures and software boundaries to provide unlimited power, collaboration and information access to everyone connected to a Grid.

In both e-business and e-science, we often need to integrate services across distributed, heterogeneous, dynamic "virtual organizations" formed from the disparate resources within a single enterprise and/or from external resource sharing and service provider relationships. This integration can be technically challenging because of the need to achieve various qualities of service when running on top of different native platforms. We present an Open Grid Services Architecture that addresses these challenges. Building on concepts and technologies from the Grid and Web services communities, this architecture defines a uniform exposed service semantics (the Grid service); defines standard mechanisms for creating, naming, and discovering transient Grid service instances; provides location transparency and multiple protocol bindings for service instances; and supports integration with underlying native platform facilities. The Open Grid Services Architecture also defines, in terms of Web Services Description Language (WSDL) interfaces and associated conventions, mechanisms required for creating and composing sophisticated distributed systems, including lifetime management, change management, and notification. Service bindings can support reliable invocation, authentication, authorization, and delegation, if required. [2,5]

The major benefits of Grids technology (like resource optimization, improved manageability and availability, capacity and disaster planning, creation of virtual organizations) introduce an important improvement versus actual distributed system technology. An increasing interest by important IT companies like IBM, Microsoft and Sun Microsystems for applications and systems, Oracle for database permits to the consideration of Grids as the future technology for distributed applications. Innovative and active service provisioning can take advantages from this technology using its features to improve quality of service. We are suggesting an extension to the actual grid solution to provide support for mobile agent activities. The platform results will be a more simple and flexible grid structure highly optimized for asynchronous and distributed processing. [6, 7]

\subsection{Mobile Data Communication}

GSM, the Global System for Mobile Communication has become the most widely used mobile communication system in the world. New bearer services have been introduced to improve GSM data communication: HSCSD, GPRS, EDGE. The last one allows operators to offer 3G services in existing frequency bands without having to purchase a $3 \mathrm{G}$ license. It provides fast connection set-up, higher bandwidth up to $150 \mathrm{Kbit} / \mathrm{s}$ and full 3GPP QoS class support.

The upcoming new $3 \mathrm{G}$ mobile network, the UMTS (Universal Mobile Telecommunications System), will boost data bit rates to up to $2 \mathrm{Mbit} / \mathrm{s}$ and will provide for a fully mobile multimedia services in a four QoS class model (conversational, streaming, interactive and background).

In the Local Area Network, IEEE802.11b [10] is the most popular Wireless LAN technology providing bandwidth up to $11 \mathrm{Mbps}$ in the band of 2,4GhZ. Powerful 5GhZ-band technologies are also already available (i.e. IEEE802.a and HiperLAN 2), providing 54Mbps of bandwidth and support for QoS (especially the latter one). While products for these technologies are already available on the market, a new standard has been introduced: the IEEE802.g that brings $54 \mathrm{Mbps}$ to $2.4 \mathrm{Ghz}$ band.

A particular mention is for Bluetooth, a wireless technology aimed as a low cost, low power, short range, and ubiquitous wireless connection. E-learning grid platform's innovative approach 
will exploit the advantages of all available technologies without being restricted to one of them and contribute to the convergence of existing access networks. In fact, these new technologies are going towards integration for both local and geographic networks, with an all IP core and with QoS support in both wired and wireless path. [11]

The assumption is that effectively these networks can offer data communication services with the needed quality of service at reasonable cost. We will use the eventually existing WLAN infrastructure set up by locally operating telecom operators; in areas not covered by WLAN the ubiquitous GPRS/UMTS-networks will be used. Low bandwidth mobile communication networks will be supported to maintain the maximum flexibility and to provide the widest connectivity to the mobile user. The envisaged applications will thus adapt to potentially limited bandwidth and changing quality of service.

\section{E-LEARNING GRID PLATFORM'S ARCHITECTURE}

The platform we propose is a new approach that will allow users to access various services and information through a variety of different end systems, including PDAs and PCs (see figure 1). The platform is based on a grid of agent environment systems that facilitate solutions for a wide range of applications and provides the following features:

- Adaptability and dynamically re-configurable.

- Resistance to breakdown.

- Support for pluggable services, applications and interfaces.

- Extendibility and Scalability.

Our solution can be shortly described using a layered model. Passing through the physical structure up to the user and service provider point of view, we can see:

- Agent Platform Infrastructure.

- Activity Management.

- Service Provisioning.

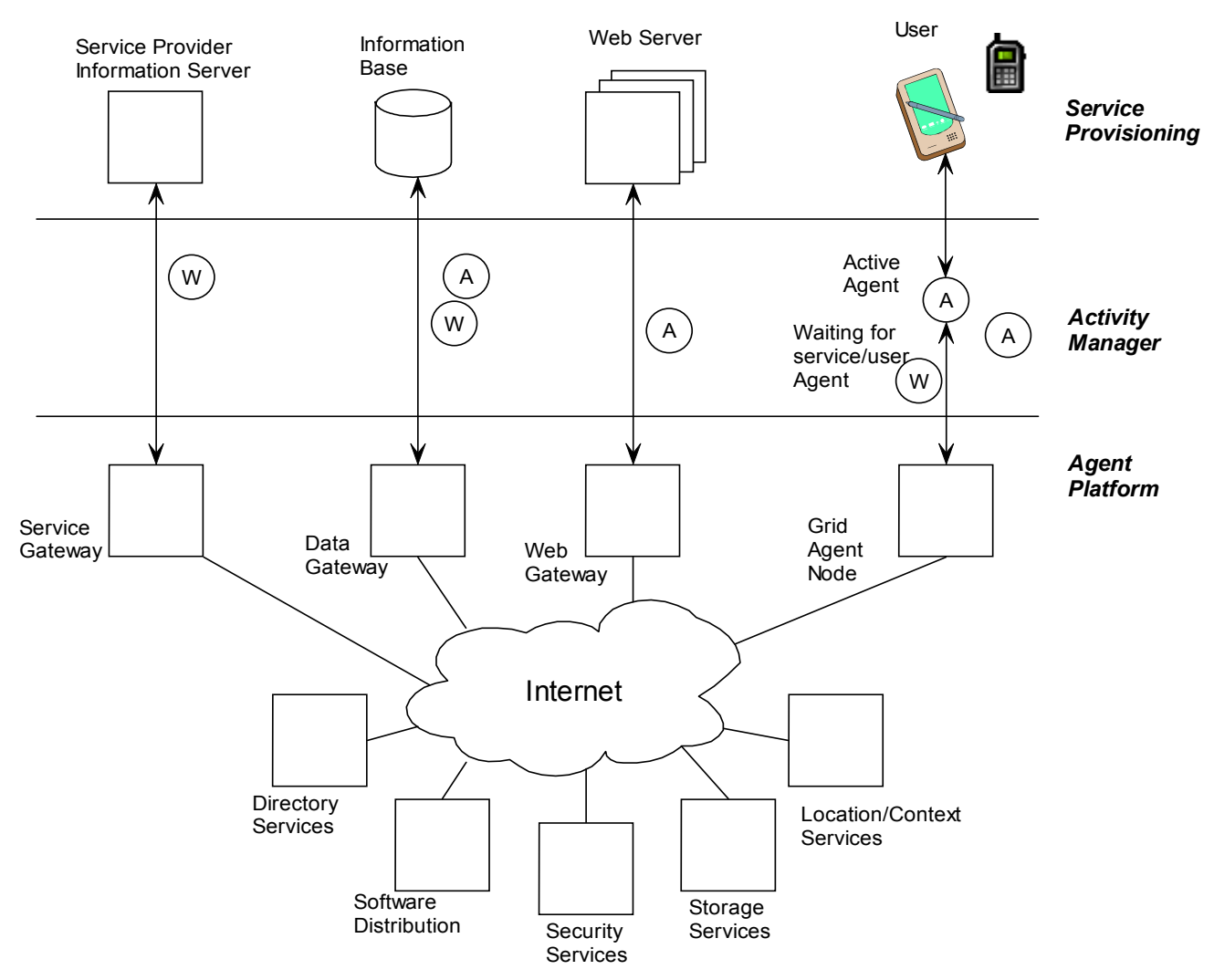

FIGURE 1:The e-learning grid platform architecture 
The Agent Platform Infrastructure represents the agents-modeled grid system upon which will be developed the basic functionalities of the system. The infrastructure includes a group of modules; each module can be installed on different systems and will work as node of a computation/storage grid sharing the load with the other available nodes. Each module will manage all the functions required for the activation and control of the mobile agents (Directory Services, Security Services, Software Distribution, Storage Services, Location/Context Services, etc.). A full package of interfaces addressed to the communication with other external systems will also be provided to open the system to provide the maximum range of the services. An important characteristic of the Agent Platform will be its ease of installation, configuration and use even by inexperienced PC users.

The Agent Platform will be implemented working with object oriented technologies (with C++ language). Agents will own dynamically defined information and will be implemented writing methods with a simple procedural language.

The internal database will be optimized for the agents' data storage and will be automatically indexed on all of the agents' public attributes without requiring any programming effort. The database will operate as a grid structure, with fault tolerance and load balance capabilities.

The end user will interact with the system by one of the supplied interfaces for PC, PDA and Mobile Phone. Over the Agent Platform will be implemented an applicative system which will allow the access to available services using the new methodology proposed for the Personal Activity Management. Each user will be able to obtain services and information simply by choosing the required service, specifying the goals to be reached and providing all the necessary information for the completion of the task. Any other interaction will be avoided and the agents system will autonomously carry out the submitted tasks returning only the final results or eventual problems encountered.

The Personal Activity Management system will be composed of a group of user interfaces, a set of infrastructure extensions and of detailed specifications.

Anyone who wishes to supply services or information will be able to use specific modules and integrate his informative system with the Agent Platform.

Activities can take on varying levels of complexity, from simple price comparisons to very complex tasks. An activity may be mapped onto actions performed by one or more agents, the results of which are then integrated by the Personal Activity Manager and presented to the user. Activities are managed by the Personal Activity Management, which also presents the results to the user. It can be used for desktop computers as well as for small handheld devices. Through a uniform yet adaptable and easy user interface the specific platform will provide access to a variety of services and applications, which can either be internal or provided by third parties.

The system presents a new methodology for creating applications - specific requirements can be accommodated through a kind of pick-and-mix approach of a number of easily configurable services. In addition to that, all users can use the system to offer services to other users. New services can easily be created and integrated into the system.

\section{CONCLUSION}

Learning, as well as other human activities, cannot be confined within well defined boundaries such as course systems. In a broader perspective, we want to enhance existing and future environments and make them more interesting from a learning perspective.

For that reason we have presented a specific e-learning platform, which is based on grid technologies. Grid technologies are getting so much noise last year, because it's a fascinating idea, which the goal is to provide unlimited power and access to vast amounts of information from any computer. The main aim of our proposed grid platform is to provide the basis for userfriendly access to information and communication services. So it will enable both business and private users, whether they are mobile or stationary, to benefit from services and applications provided through the internet. 


\section{REFERENCES}

[1] Dongarra, J., Johnson, C. and Miller, M. (1999) Application-Specific Tools. In Foster, I. and Kesselman, C. eds. The Grid: Blueprint for a New Computing Infrastructure, Morgan Kaufmann, pp 159-180.

[2] Fitzgerald, S., Foster, I. and Kesselman, C., (2001), Grid Information Services for Distributed Resource Sharing. In 10th IEEE International Symposium on High Performance Distributed Computing, IEEE Press, pp 181-184

[3] De Roure, D., Jennings, N. and Shadbolt, N. Research Agenda for the Semantic Grid: A Future e-Science Infrastructure. UK National eScience Center. www.semanticgrid.org.

[4] Foster, I. and Kesselman, C. Globus: (1999) A Toolkit-Based Grid Architecture. In Foster, I. and Kesselman, C. eds. The Grid: Blueprint for a New Computing Infrastructure, Morgan Kaufmann, pp 259-278.

[5] Foster, I. and Kesselman, C. (eds.). (1999) The Grid: Blueprint for a New Computing Infrastructure. Morgan Kaufmann.

[6] Ian Foster, Carl Kesselman, Jeffrey M. Nick, Steven Tuecke. The Physiology of Grid. An Open Grid Services Architecture for Distributed Systems Integration

[7] Stephen Shankland. Grid computing earns a living. http://zdnet.com.com/2100-110341810.html

[8] http://www-1.ibm.com/grid

[9] Graham, S., Simeonov, S., Boubez, T., Daniels, G., Davis, D., Nakamura, Y. and Neyama, R. 2001 Building Web Services with Java: Making Sense of XML, SOAP, WSDL, and UDDI.

Sams.

[10] Borisov, N., Goldberg, I., \& Wagner, D. (2001). Intercepting Mobile Communications: The Insecurity of 802.11. Retrieved September 21, 2001 from the World Wide Web http://www.isaac.cs.berkeley.edu/isaac/mobicom.pdf

[11] Muller, M. J. (2001) Bluetooth Demystified. USA: McGraw-Hill.

[12] http://www.blueu.com/elearn/why_el.html 